\title{
$\nabla$
}

\section{Atypical presentation of histoplasmosis in an immunocompromised patient*}

\author{
Talita voss Gonzalez ${ }^{1}$ \\ Angelo Sementilli ${ }^{2}$ \\ Patrícia Mara Beltrame ${ }^{3}$
}

\author{
Sandra Lopes Mattos e Dinato ${ }^{2}$ \\ Ney Romiti (In memoriam) ${ }^{2}$ \\ Ana Paula Rocha Veiga ${ }^{2}$
}

DOI: http:/ / dx.doi.org/10.1590/abd1806-4841.20153782

\begin{abstract}
A bstract: We present a case of disseminated cutaneous histoplasmosis in a male patient, rural worker, HIV positive for 20 years, with a history of irregular use of antiretroviral therapy, T cell counts below 50 cells/mm 3 and with good response to treatment with Itraconazole. We highlight importance of skin lesions in clarifying early diagnosis, since this co-infection often leads patients to death.
\end{abstract}

Keywords: HIV; HIV infections; Histoplasmosis

\section{INIRODUCTION}

Histoplasmosis is a systemic mycosis caused by a dimorphic fungus, $\mathrm{H}$ istoplasma capsulatum var capsulatum, which is endemic in Latin America and other tropical countries. ${ }^{1}$ It is a saprophytic fungus found in soil contaminated by birds feces. Primary infection is acquired through inhalation of conidia present in nature (caves with bats, chicken coops, etc.). It is a self-limited disease with clinical signs absent in healthy individuals. ${ }^{2}$ In individuals exposed to large numbers of spores, late cavitary pulmonary histoplasmosis, granulomatous mediastinitis or mediastinal fibrosis may occur. ${ }^{3}$

Clinical presentations are: acute and chronic pulmonary histoplasmosis, disseminated and primary cutaneous ${ }^{2}$. Infection is limited and restricted to the lungs in $99 \%$ of cases; the rest progresses to disseminated or chronic form. Advanced disease may occur as a progression of acute infection or late reactivation of focus with viable fungi. ${ }^{4}$ In the disseminated form, the main findings are weight loss, fever, hepatosplenomegaly, generalized lymphadenopathy, and involvement of bone marrow, CNS, skin and mucous membranes. Regarding skin lesions, they are generally nonspecific, manifesting as macules, papules, pustules, verrucous plaques and ulcers. ${ }^{2}$ Ulcers may also be found on oral and labial mucosa. ${ }^{5}$
In immunocompromised subjects, particularly in AIDS patients when their CD4 T-lymphocyte count is less than 50 cells $/ \mathrm{mm} 3$, histoplasmosis may be presented in the form of a severe and widespread infection. ${ }^{5}$ Skin lesions occur in $4-11 \%$ of patients and result from secondary invasion of skin in widespread form of infection, and it may be the first sign of the disease in immunocompromised individuals. ${ }^{6}$

The gold standard for diagnosis is the anatomopathological examination and culture for fungi of the involved skin tissues. ${ }^{2}$ Fungus may also be evidenced in the sputum, blood, bone marrow and urine sediment. ${ }^{2}$ Histopathological examination can detect the fungus through PAS and Grocott stains. The material for culture can be obtained by biopsy, aspiration, bronchial lavage and blood or marrow punction. ${ }^{2}$ Detection of polysaccharide antigens in fluids such as urine or serum are also helpful, but there may be false-positive results, especially in patients with paracoccidioidomycosis. ${ }^{3}$

Complement fixation and immunodiffusion tests are usually negative in patients with HIV. ${ }^{3}$ PCR on samples of blood and tissue is highly sensitive and specific. Histoplasmin skin test is indicated for non-endemic areas. $^{2}$

\section{Received on 18.06.2014}

Approved by the Advisory Board and accepted for publication on 11.05.2014

Study performed at Centro Universitário Lusíada (UNILUS) and Hospital Guilherme Álvaro- Santos (SP), Brazil.

Financial Support: None.

Conflict of Interest: None.

Irmandade da Santa Casa de Misericórdia de Santos (SCMS) - Santos (SP), Brasil.

Centro Universitário Lusíada (UNILUS)- Santos (SP), Brasil.

Hospital Guilherme Álvaro - Santos (SP), Brasil.

C2015 by Anais Brasileiros de Dermatologia 
The objective of this study is to report the case of a subject with large skin and mucosa lesions, which allowed the diagnosis and early treatment of the disease. The patient was infected with HIV for 20 years and had severe immunosuppression, with disseminated fungal disease and satisfactory clinical outcome despite the severity of co-infection.

\section{CASE REPORT}

Man, 52 years old, rural worker, infected with $\mathrm{HIV}$, with severe immunosuppression due to the irregular treatment with antiretroviral drugs. He has presented for 2 months poor general condition and verrucous lesions throughout integument, predominantly in the face and trunk (Figure 1). Examination showed pancytopenia and renal dysfunction (creatinine clearance $27 \mathrm{ml} / \mathrm{min}$ ). Chest tomography revealed diffuse accentuation of the lung interstitium. Pathological examination (HE) showed epidermis with central ulceration, necrosis and numerous macrophages with clear cytoplasm and oval structures (Figure 2). Grocott stain was positive for Histoplasma (Figure 3). Direct mycological examination revealed fungal structures compatible with Histoplasma. Culture was performed from skin biopsy sample in Sabouraud-dextrose agar, at room temperature. On macroscopic examination white cotton-wool spots colonies were observed and, on microscopic examination, presence of hyphae, with rounded microconidia and macroconidia, was noted (Figure 4 and 5). Myeloculture and blood culture for fungus also showed the presence of $\mathrm{H}$ istoplasma capsulatum var capsulatum. Serology for specific antibodies was negative for the fungus. During hospitalization, the patient developed febrile neutropenia and therapy with Cefepime, Granulokine and reintroduction of antiretrovirals was started. CD4 was $7 \mathrm{cel} / \mathrm{m}^{3}$ and viral

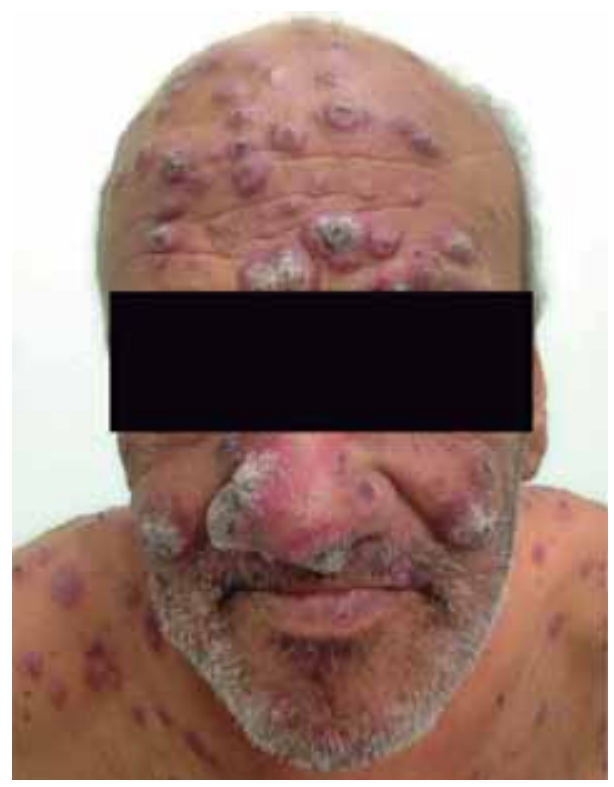

Figure 1:

Pili torti. Polarized light microscopy, $10 \mathrm{x}$ magnification: Hair twisted about its longitudinal axis

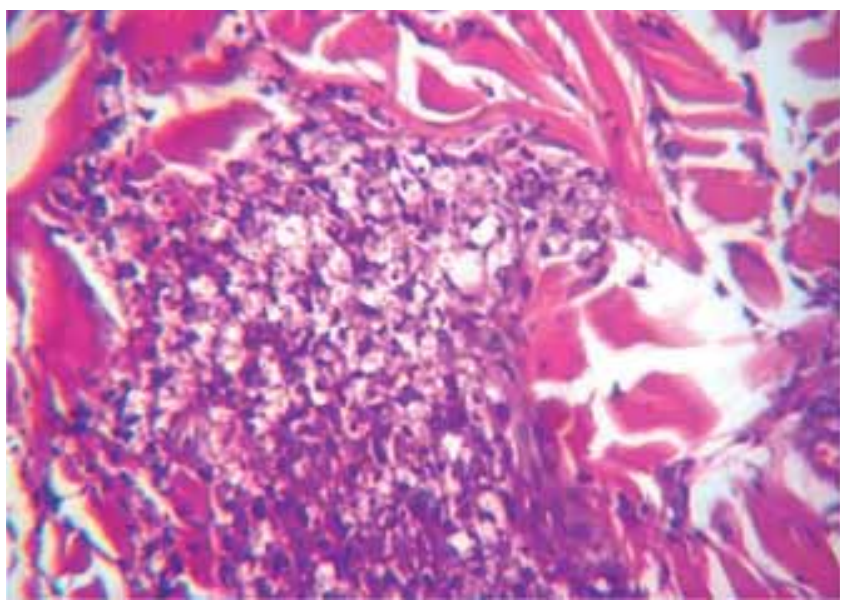

Figure 2: 100X HE: Skin sample containing histiocytic infiltration in the dermis, which show punctate structures in the cytoplasm

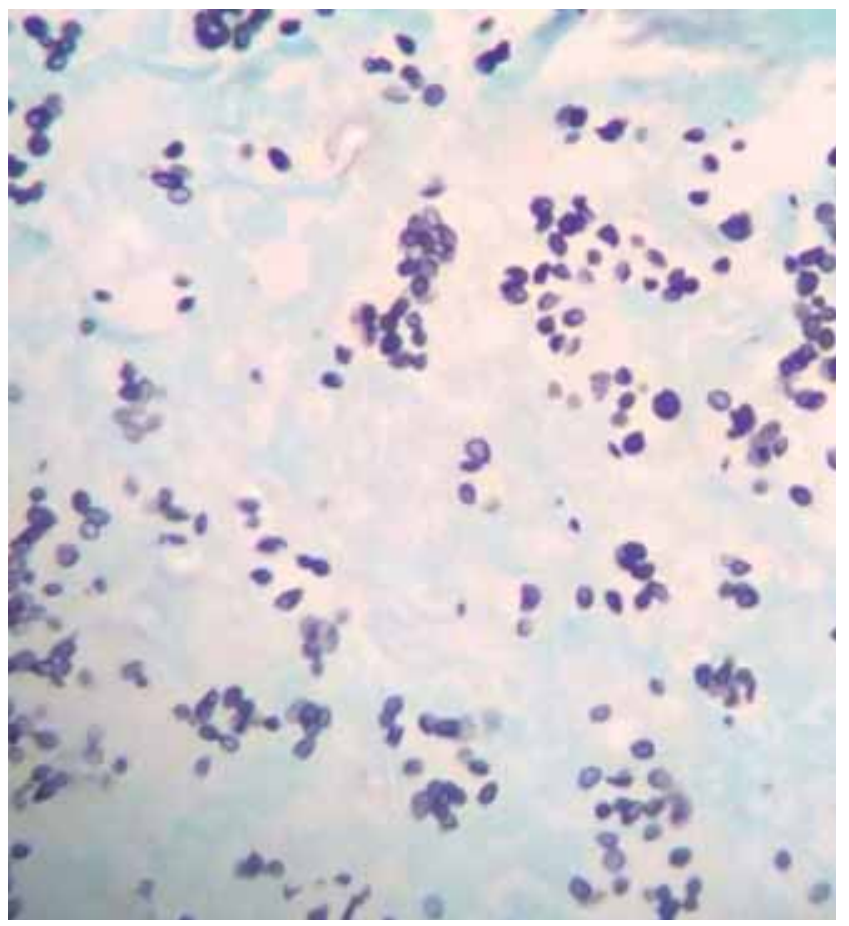

Figure 3: Groccot 400X: In the silver staining it's possible to note oval and uniform intracellular and grouped structures compatible with H istoplasma

load was 598,342 copies. A chemoprophylaxis with trimethoprim-sulfamethoxazole and azithromycin was performed. Therapy adopted was Itraconazole - 200 mg 3 times daily for 3 days in a row followed by 400 $\mathrm{mg} /$ day. It was of utmost importance the reintroduction of antiretrovirals, which led to an CD4 increase.

The evolution of the case was satisfactory, with progressive improvement soon after the institution of specific antifungal treatment. After monitoring for 11 months, the patient had a weight gain of 20 kilos, clinical resolution of all lesions and improvement in general condition (Figure 6). 


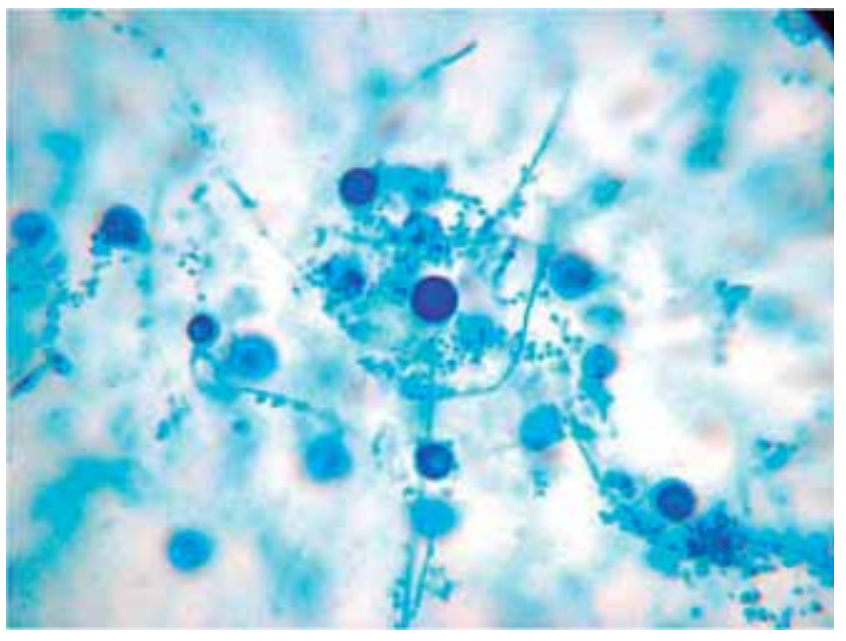

FIGURE 4: Culture - Microscopic examination: presence of tuberculate microconidia and macroconidia

\section{DISCUSSION}

The most important risk factor for reactivation of infection and progression to disease is HIV-induced immunosuppression. ${ }^{3}$

Co-infection of histoplasmosis and AIDS, particularly when CD4 T-lymphocytes count is less than 50 cells $/ \mathrm{mm} 3$, leads to a severe and disseminated infection. ${ }^{5}$ In our case, the importance of skin lesions as a sign of systemic disease was evident, and it also may assist in the early diagnosis of histoplasmosis. This allowed the immediate start of specific treatment, thus reducing the risk of disease progression, which has a high mortality rate in immunocompromised patients. ${ }^{3}$

Skin lesions are present in most Brazilian cases (38-85\%) and they are often more extensive compared

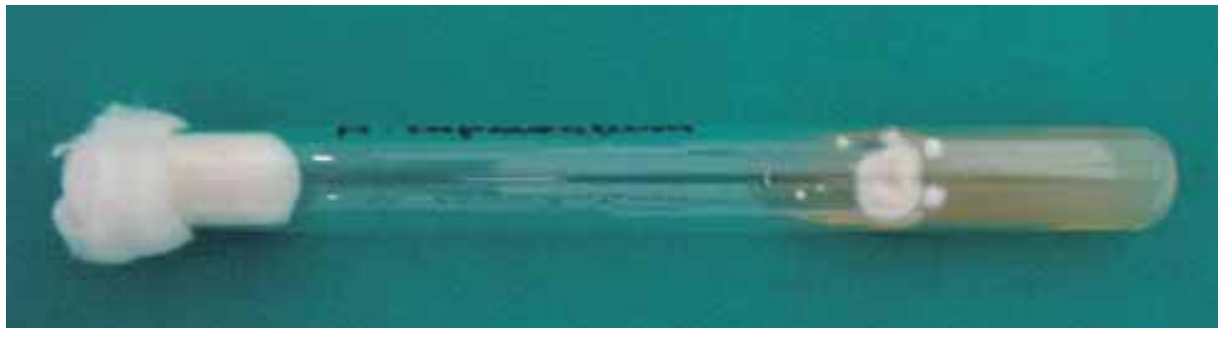

Figure 5:

Culture - macroscopic examination: presence of white cotton-wool spots nies

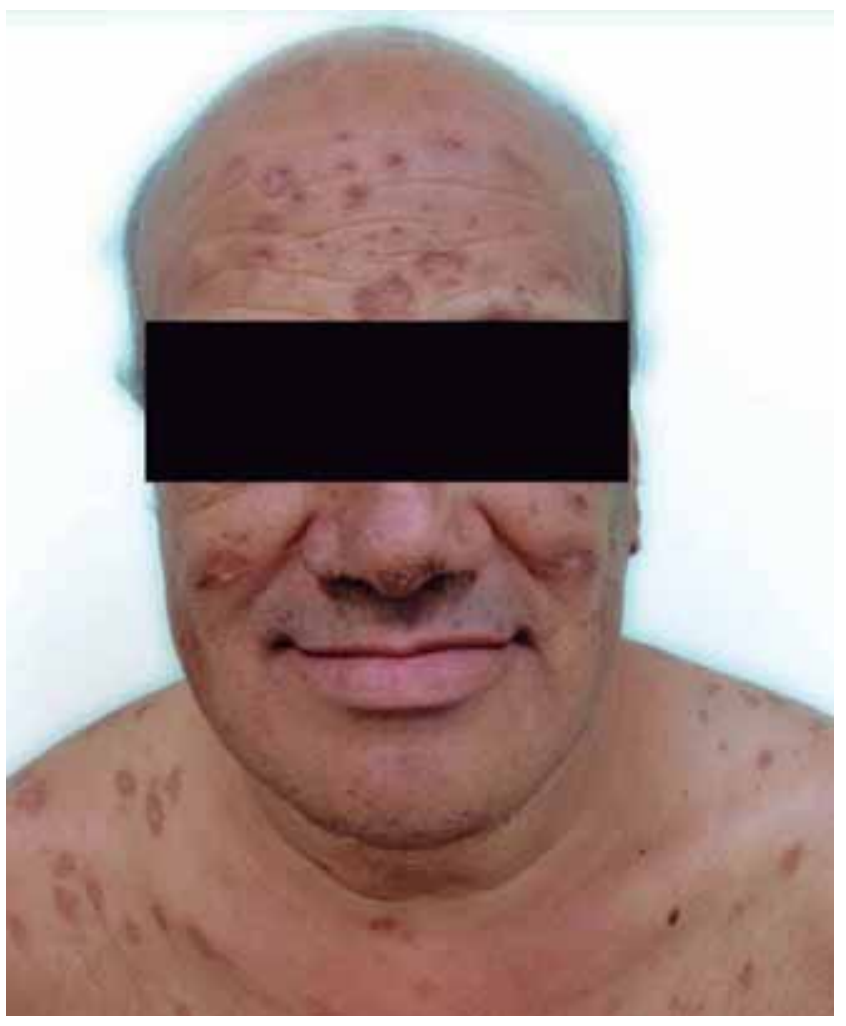

FigurE 6: Culture - macroscopic examination: presence of white cotton-wool spots colonies with those reported in the USA, thus justifying the tropism for skin of strains diagnosed in South America. ${ }^{3}$ Skin lesions predominate when $\mathrm{H}$. duboisii is the etiologic agent. ${ }^{7}$ Histoplasmosis mortality in immunosuppressed patients is greater than $33 \%$, while in immunocompetent individuals is approximately $17 \%{ }^{3}$

In the literature, the drug of choice for disseminated histoplasmosis is Amphotericin B, $0.5-0.7 \mathrm{mg} /$ $\mathrm{kg} /$ day for 10 weeks, followed by Itraconazole, 200 $\mathrm{mg} 3$ times a day and maintenance dose of $400 \mathrm{mg} /$ day for 12 weeks. ${ }^{8-9}$

For our patient, the treatment of choice was Itraconazole, due to the associated comorbidities. The patient presented excellent therapeutic response, and the drug was kept at a dose of $400 \mathrm{mg}$ /day until reversal of immunosuppression state. The patient remains well during outpatient follow up, already for 11 months. In conclusion, it should be emphasized that histoplasmosis, particularly in HIV-infected patients, needs early diagnostic clarification, seeking immediate specific therapy, which will surely bring better progress. $\square$ 


\section{REFERENCES}

1. Vasudevan B, Ashish B, Amitabh S, A P M. Primary cutaneous histoplasmosis in a HIV-positive individual. J Glob Infect Dis. 2010;2:112-5.

2. Kauls $L$, Blauvelt $A$, Skin disease in acute and chronic immunosuppression. In: Wolff K, Goldsmith LA, Katz SI, Gilchrest BA, Paller AS, Leffell DJ, editors. Fitzpatrick's Dermatology in General Medicine. 7th ed. New York: McGraw Hill; 2008. p. $275-6$.

3. Cunha VS, Zampese MS, Aquino VR, Cestari TF, Goldani LZ. Mucocutaneous manifestations of disseminated histoplasmosis in patients with acquired immunodeficiency syndrome: particular aspects in a Latin-American population. Clin Exp Dermatol. 2007;32:250-5.

4. Kauffman CA. Histoplasmosis: a clinical and laboratory update. Clin Microbiol Rev. 2007;20:115-32.

5. Orsi AT, Nogueira L, Chrusciak-Talhari A, Santos M, Ferreira LC, Talhari S, et al. Histoplasmosis and AIDS co-infection. An Bras Dermatol. 2011;86:1025-6.

6. Saeki NM, Schubach A0, Salgueiro MM, Silva CF, et al. Histoplasmose cutânea primária: relato de caso em paciente imunocompetente e revisão de literatura. Rev Soc Bras Med Trop. 2008;41:680-2.

7. Burns T, Breathnach S, Cox N, Griffiths C. Rook's Textbook of Dermatology 8th ed. Oxford: Wiley-Blackwell Publishing Ltd; 2010. p 36.82-84

8. Wheat LJ, Freifeld AG, Kleiman MB, Baddley JW, McKinsey DS, Loyd JE, et al. Clinical practice guidelines for management of patients with histoplasmosis:2007 update by the infectious Disease of America. Clin Infect Dis. 2007;45:807-25.

9. Bhagwat PV, Hanumanthayya K, Tophakhane RS, Rathod RM. Two unusual cases of histoplasmosis in human immunodeficiency virus-infected individuals. Indian J Dermatol Venereol Leprol. 2009;75:173-6.

M AILING ADDRESS:

Talita voss Gonzalez

197, D r O swaldo Cruz Street, Boqueirão

11045904 - Santos - SP

Brazil

Email: talita.voss@hotmail.com

H ow to cite this article: voss Gonzalez T, Mattos e Dinato SL, Sementilli A, Romiti N, Beltrame PM, Veiga APR. Atypical presentation of histoplasmosis in na immunocompromised patient.. An Bras Dermatol. 2015;90 (3 Suppl 1): S32-5. 